\title{
Numerical simulation of travelling wave induced electrothermal fluid flow
}

\author{
Ivan R Perch-Nielsen ${ }^{1}$, Nicolas G Green ${ }^{2}$ and Anders Wolff ${ }^{1}$ \\ ${ }^{1}$ Department of Micro and Nanotechnology (MIC), Technical University of Denmark \\ (DTU), Building 345 east, DK-2800 Kongens Lyngby, Denmark \\ ${ }^{2}$ School of Electronics and Computer Science, University of Southampton, SO17 1BJ, UK \\ E-mail:ng2@ecs.soton.ac.uk
}

Received 10 March 2004

Published 28 July 2004

Online at stacks.iop.org/JPhysD/37/2323

doi:10.1088/0022-3727/37/16/016

\begin{abstract}
Many microdevices for manipulating particles and cells use electric fields to produce a motive force on the particles. The movement of particles in non-uniform electric fields is called dielectrophoresis, and the usual method of applying this effect is to pass the particle suspension over a microelectrode structure. If the suspension has a noticeable conductivity, one important side effect is that the electric field drives a substantial conduction current through the fluid, causing localized Joule-heating. The resulting thermal gradient produces local conductivity and permittivity changes in the fluid. Dielectrophoretic forces acting upon these pockets of fluid will then produce motion of both the fluid and the particles.

This paper presents a numerical solution of the electrical force and the resulting electrothermal driven fluid flow on a travelling wave structure. This common electrode geometry consists of interdigitated electrodes laid down in a long array, with the phase of the applied potential shifted by $90^{\circ}$ on each subsequent electrode. The resulting travelling electric field was simulated and the thermal field and electrical body force on the fluid calculated, for devices constructed from two typical materials: silicon and glass. The electrothermal fluid flow in the electrolyte over the electrode array was then numerically simulated.

The model predicts that the thermal field depends on the conductivity and applied voltage, but more importantly on the geometry of the system and the material used in the construction of the device. The velocity of the fluid flow depends critically on the same parameters, with slight differences in the thermal field for glass and silicon leading to diametrically opposite flow direction with respect to the travelling field for the two materials. In addition, the imposition of slight external temperature gradients is shown to have a large effect on the fluid flow in the device, under certain conditions leading to a reversal of the fluid flow direction.
\end{abstract}

\section{Introduction}

Cell sorting requires an effective method of discrimination. Conventionally, this has been done by labelling the cells with fluorescent antibodies and sorting them using a fluorescent activated cell sort (FACS) [1]. Another method that is increasingly used is the manipulation of cells using electric fields, a technique that is non-contact and does not require an alteration of the cells. When an electric field is applied to particles such as biological cells, a dipole is induced. If the electric field is non-uniform, one half of the dipole will experience a stronger field than the other, giving rise to a net force on the particle. This type of electric field induced particle motion is known as dielectrophoresis (DEP) [2-4].

The frequency dependent DEP force on a cell depends on the internal properties and structure of the cell. It is therefore possible to discriminate between cell-types and their properties without the need for chemical labelling, and several designs 


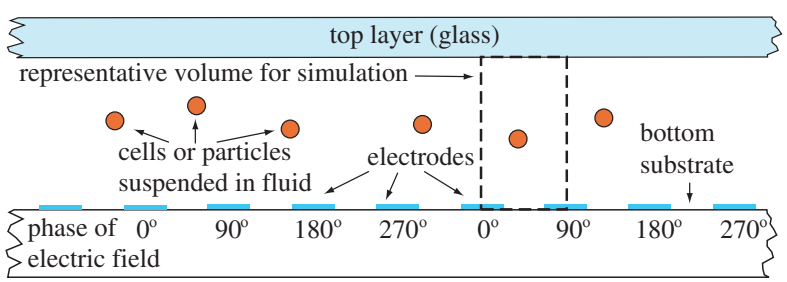

Figure 1. Schematic side view of the travelling wave electrode configuration, with successive coplanar, long, narrow plate electrodes arranged parallel to each other and fabricated on a planar substrate (glass or silicon). The conducting fluid (usually a solution of ions in water) is passed through a microchannel constructed over the array, in contact with the electrodes. The electrodes are energized using ac potential signals with relative phases arranged with successive $90^{\circ}$ phase shifts as shown. The box indicated by the dashed line is the minimum representative volume that must be solved in the simulations to give complete information about the whole array.

(This figure is in colour only in the electronic version)

of electrode arrays have been used for this [5]. One particular technique, travelling wave DEP (twDEP) [5,6], uses variations of the type of electrode array shown in figure 1. The twDEP structure in its basic and most widely used design consists of an array of parallel electrodes which are coplanar on a planar substrate, have a regular spacing and width and are much longer across the array than their width (in the direction along the array and the channel). AC potentials are applied to the electrodes with the phase shifted by $90^{\circ}$ on each subsequent electrode. In this type of field, the spatially dependent phase of the field and the phase-lag between the induced dipole and the field results in an additional component of the DEP force [3].

Calculating the electric field induced force on the particle and its ensuing motion is relatively simple [3]. However, for DEP electrode arrays in general, the electric field not only produces an induced particle motion but is also responsible for an induced fluid flow in the suspending medium [7-10]. There are several physical mechanisms whereby the electric field can interact with the fluid, ranging from bulk effects such as convection and electrothermal effects to surface effects such as ac electro-osmosis [10]. These effects are constantly present and have a large variation in magnitude, ranging from a value that is negligible in comparison to the particle velocity, to one which dominates the system completely. These flow phenomena are normally neglected as they are difficult to model and verify experimentally. However, when the frequency equals the charge relaxation frequency of the media [11] the fluid flow will dominate the movement of particles, and a more thorough understanding is useful.

This paper presents the results of a numerical simulation of the electrothermal driven fluid flow caused by Joule heating in the generic twDEP structure shown in figure 1. Only the effect caused by Joule heating is modelled, as ac electroosmosis and buoyancy are several orders of magnitude smaller in the specific system [11]. The modelled structure has an electrode width and spacing of $10 \mu \mathrm{m}$. Two different devices have been simulated, one with a glass substrate, the other with silicon. Both devices have the same dimensions, sizes and electrode geometry, the only difference being the thermal and electrical conductivity of the substrate. The different materials and external temperature gradients are demonstrated to have a critical influence on the electrothermal driven fluid flow, including a complete reversal of direction relative to the field under a range of conditions. The simulation is carried out in three steps: first the electrical field from the electrodes is calculated; second, the heat-distribution through the fluid and the device as a whole is calculated; and third, the electrothermal body force, determined from the thermal and electric fields, is used as the body force in the Navier-Stokes equation to determine the resulting fluid velocity.

\section{Theory}

When an electric field is applied to a conducting fluid, an electrical current passes through it, locally generating heat proportional to the conductivity and the field strength squared (Joule heating) [12]. Since the field is non-uniform, the heat generation is non-uniform and the diffusion of heat through the system is rapid, leading to a static thermal field [10]. The variation in temperature through the fluid leads to variations in the local conductivity $(\sigma)$ and permittivity $(\varepsilon)$ of the fluid. Where there are gradients in $\sigma$ and $\varepsilon$ an applied electric field will impose an electric force on the fluid [13].

\subsection{The electric field}

In this paper only time-averaged forces and flows are required as opposed to instantaneous values. As a result of this and the fact that the field is harmonic, phasor notation can be used. The phasor includes the spatial variation of both the magnitude and phase of the electric field. In phasor notation, a potential oscillating at frequency $\omega$ can be defined

$$
\phi(x, t)=\operatorname{Re}\left[\tilde{\phi}(\boldsymbol{x}) \mathrm{e}^{\mathrm{i} \omega t}\right],
$$

where $\boldsymbol{x}$ is the position vector, $\omega$ the angular frequency, $t$ the time and $\tilde{\phi}=\phi_{\mathrm{r}}+\mathrm{i} \phi_{\mathrm{i}}$ is the potential phasor, with $\phi_{\mathrm{r}}$ the real and $\phi_{\mathrm{i}}$ the imaginary part. The Laplace equation is then solved for the potential phasor [4]:

$$
\nabla^{2} \tilde{\phi}=0 .
$$

The electric field phasor, $\tilde{\boldsymbol{E}}=\boldsymbol{E}_{\mathrm{r}}+\mathrm{i} \boldsymbol{E}_{\mathrm{i}}$, can then be found from the potential:

$$
\tilde{\boldsymbol{E}}=-\nabla \tilde{\phi}=-\left(\nabla \phi_{\mathrm{r}}+\mathrm{i} \nabla \phi_{\mathrm{i}}\right) .
$$

\subsection{The thermal field}

As discussed above, the strong electric fields in a microstructure give rise to Joule heating in the fluid. The time average of the power dissipated per unit volume can be written as

$$
\langle W\rangle=\frac{1}{2}\left\langle\sigma E^{2}\right\rangle .
$$

$\sigma$ is the electrical conductivity and $E=\|\tilde{\boldsymbol{E}}\|$ is the electric field strength at the point of interest. To find the temperature $T$, the energy balance equation must be solved $[14,15]$, which, as discussed in [10], reduces to the heat diffusion equation

$$
k \nabla^{2} T+\frac{1}{2}\left\langle\sigma E^{2}\right\rangle=0,
$$

where $k$ is the thermal conductivity. This must be solved over the whole system (substrate, fluid and glass-lid) to give the correct thermal field. 


\subsection{The electric force density and resulting fluid flow}

When the electric field and the temperature profile have been simulated, the resulting force on the fluid due to Joule heating can be calculated, with the time averaged electric force density being given by [10]

$\left\langle\boldsymbol{f}_{\mathrm{e}}\right\rangle=\frac{1}{2} \operatorname{Re}\left[\left(\frac{(\sigma \nabla \varepsilon-\varepsilon \nabla \sigma) \cdot \tilde{\boldsymbol{E}}}{\sigma+\mathrm{i} \omega \varepsilon}\right) \tilde{\boldsymbol{E}}^{*}-\frac{1}{2}|\tilde{\boldsymbol{E}}|^{2} \nabla \varepsilon\right]$.

For small changes in temperature, the changes in the permittivity and conductivity of the fluid can be written as $\nabla \varepsilon=(\partial \varepsilon / \partial T) \nabla T$ and $\nabla \sigma=(\partial \sigma / \partial T) \nabla T$. Substituting these and setting $\alpha=(\partial \varepsilon / \partial T) / \varepsilon$ and $\beta=(\partial \sigma / \partial T) / \sigma$, equation (6) can be rewritten as $[4,16]$

$\left\langle\boldsymbol{f}_{\mathrm{e}}\right\rangle=\frac{1}{2} \operatorname{Re}\left[\frac{\sigma \varepsilon(\alpha-\beta)}{\sigma+\mathrm{i} \omega \varepsilon}(\nabla T \cdot \tilde{\boldsymbol{E}}) \tilde{\boldsymbol{E}}^{*}-\frac{1}{2} \varepsilon \alpha|\tilde{\boldsymbol{E}}|^{2} \nabla T\right]$.

For aqueous $\mathrm{KCl}$ solutions of moderate concentration, $\alpha=$ $-0.4 \% \mathrm{~K}^{-1}, \beta=2.0 \% \mathrm{~K}^{-1}$ [17] and $\varepsilon=80 \varepsilon_{0}$ where $\varepsilon_{0}=8.854 \times 10^{-12} \mathrm{Fm}^{-1}$. The conductivity, $\sigma$, can be changed easily, e.g. by adjusting the concentration of $\mathrm{KCl}$ with the values used in this paper varying from $1 \times 10^{-3} \mathrm{~S} \mathrm{~m}^{-1}$ to $1 \mathrm{~S} \mathrm{~m}^{-1}$. Equation (7) can be separated into real and imaginary components of the field phasor: $\boldsymbol{E}_{\mathrm{r}}=\operatorname{Re}[\tilde{\boldsymbol{E}}]$ and $\boldsymbol{E}_{\mathrm{i}}=\operatorname{Im}[\tilde{\boldsymbol{E}}]$, so that

$$
\begin{aligned}
\left\langle\boldsymbol{f}_{\mathrm{e}}\right\rangle= & \frac{1}{2}\left[\frac { \varepsilon ( \alpha - \beta ) } { 1 + ( \omega \varepsilon / \sigma ) ^ { 2 } } \left\{\left(\nabla T \cdot \boldsymbol{E}_{\mathrm{r}}\right) \boldsymbol{E}_{\mathrm{r}}+\left(\nabla T \cdot \boldsymbol{E}_{\mathrm{i}}\right) \boldsymbol{E}_{\mathrm{i}}\right.\right. \\
& \left.\left.+\frac{\omega \varepsilon}{\sigma}\left[\left(\nabla T \cdot \boldsymbol{E}_{\mathrm{r}}\right) \boldsymbol{E}_{\mathrm{i}}-\left(\nabla T \cdot \boldsymbol{E}_{\mathrm{i}}\right) \boldsymbol{E}_{\mathrm{r}}\right]\right\}\right] \\
& -\frac{1}{4} \varepsilon \alpha \nabla T\left[\left|\boldsymbol{E}_{\mathrm{r}}\right|^{2}+\left|\boldsymbol{E}_{\mathrm{i}}\right|^{2}\right] .
\end{aligned}
$$

The steady state velocity of the fluid produced by the electrothermal body force can then be calculated from the Navier-Stokes equation [18] which, for low Reynolds number in an incompressible fluid, is simplified to

$$
-\nabla p+\eta \nabla^{2} \boldsymbol{u}+\left\langle\boldsymbol{f}_{\mathrm{e}}\right\rangle=0
$$

where $p$ is the pressure, $\eta$ the viscosity and $\boldsymbol{u}$ is the fluid velocity.

\section{Numerical model and boundary conditions}

We used FlexPDE ${ }^{\mathrm{TM}}$, a partial differential equation solver [19], to obtain a numerical solution of the equations. The simulation was carried out in three steps: first the electric field was calculated, then the resulting thermal field, and finally the Navier-Stokes equation was solved to give the fluid velocity.

The simulated structure has a bottom substrate thickness of $1000 \mu \mathrm{m}$ and consists of two materials: silicon and glass. A $2 \mu \mathrm{m}$ silicon nitride layer is deposited on top of the silicon substrate as insulation for the electrodes. On top of the substrate, there is the twDEP electrode structure, which consists of a repeated set of four $10 \mu \mathrm{m}$ electrodes with a thickness of $100 \mathrm{~nm}$, separated by a $10 \mu \mathrm{m}$ gap. Above the electrodes is a $100 \mu \mathrm{m}$ layer of fluid and finally a lid of $100 \mu \mathrm{m}$ glass.
Even though there are 4 electrodes with different phases applied, there are several symmetries that can be used to reduce the problem. The minimum solution space is a vertical section from the centre of the first electrode to the centre of the next [20] (figure 2). The permittivity for glass is $\varepsilon_{\mathrm{G}}=3 \varepsilon_{0}$ and the conductivity is negligible. The values for silicon are $\varepsilon_{\mathrm{Si}}=12 \varepsilon_{0}$ and $\sigma_{\mathrm{Si}}=5-100 \mathrm{kS} \mathrm{m}^{-1}$. The conductivity of the fluid was varied from $\sigma=1 \times 10^{-3}$ to $1 \mathrm{~S} \mathrm{~m}^{-1}$.

\subsection{Electrical model}

In order to model the complex field, the electric potential is solved for a set of boundary conditions that represents the phasor across the electrode array. The values of the boundary conditions for the real $\left(\phi_{\mathrm{r}}\right)$ and imaginary $\left(\phi_{\mathrm{i}}\right)$ parts of the potential are shown in figure $2(a)$. Since the potential at the phase $90^{\circ}$ electrode is 0 , and the potentials on the electrodes with phases $0^{\circ}$ and $180^{\circ}$ have opposite signs, the real parts of the potentials on the vertical lines through the centres of the $90^{\circ}$ and $270^{\circ}$ electrodes are zero. The real parts of the potentials on the vertical lines through the centres of the $0^{\circ}$ and $180^{\circ}$ electrodes have even symmetry, giving the boundary condition $\partial \phi_{\mathrm{r}} / \partial n=0$. The same arguments hold true for the imaginary parts, but shifted along one electrode.

On the interfaces between the water and substrate, the electric field must satisfy the condition

$$
\left(\sigma_{\mathrm{w}}+\mathrm{i} \omega \varepsilon_{\mathrm{w}}\right) \tilde{\boldsymbol{E}}_{\mathrm{w}} \cdot \hat{\boldsymbol{n}}=\left(\sigma_{\mathrm{s}}+\mathrm{i} \omega \varepsilon_{\mathrm{s}}\right) \tilde{\boldsymbol{E}}_{\mathrm{s}} \cdot \hat{\boldsymbol{n}},
$$

where $\hat{\boldsymbol{n}}$ is the normal to the boundary surface, in this case directed from substrate to water. Since the conductivity and permittivity of water are large compared to glass (for glass substrate) and the insulating layer between the electrodes and silicon (for silicon substrate), the right-hand side of equation (10) can be set to zero. This, together with equation (3), gives the condition $\partial \tilde{\phi} / \partial n=0$ on the lower boundary. Finally, the electrodes are much longer than the width, so the $z$-plane can be ignored, reducing the problem to a two-dimensional one.

The electrode thickness $(100 \mathrm{~nm})$ is small compared to the width, so they can be considered infinitely thin and are therefore not included as objects. The field from the electrodes are added by giving the solver a fixed value for the potential along the lower boundary.

\subsection{Thermal model}

For the simulations, the thermal conductivity $(k)$ used for glass was $1 \mathrm{WK}^{-1} \mathrm{~m}^{-1}$, silicon was $140 \mathrm{WK}^{-1} \mathrm{~m}^{-1}$ (more than two orders of magnitude higher) and for water was $k_{\mathrm{w}}=0.6 \mathrm{WK}^{-1} \mathrm{~m}^{-1}$. As seen from equation (4), only the absolute value of the electric field is used for finding the temperature distribution. Since $\boldsymbol{E}_{\mathrm{r}}$ and $\boldsymbol{E}_{\mathrm{i}}$ will be mirror images of each other, the absolute value will be symmetrical above each electrode. This will be reflected in the temperature distribution, and the left and right boundaries of the thermal model, therefore, have the condition $\partial T / \partial n=0$. Lastly, since the electrodes are thin, compared to the geometry of the structure, and their thermal conductivity is significantly higher than the rest of the system, heat can be assumed to conduct instantaneously from the fluid to the substrate through 
(a)

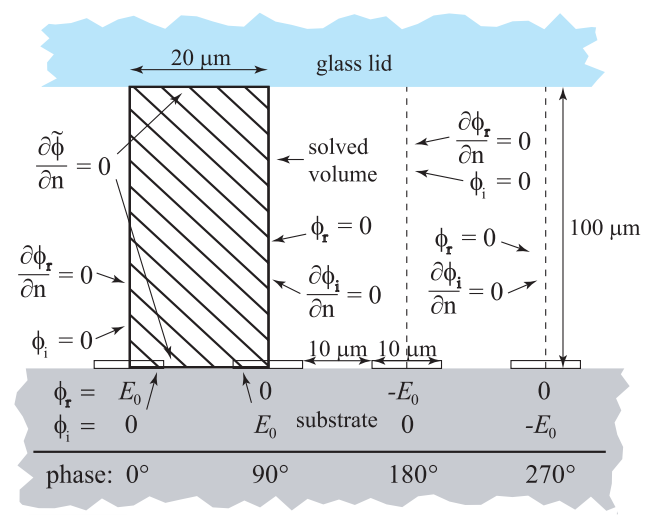

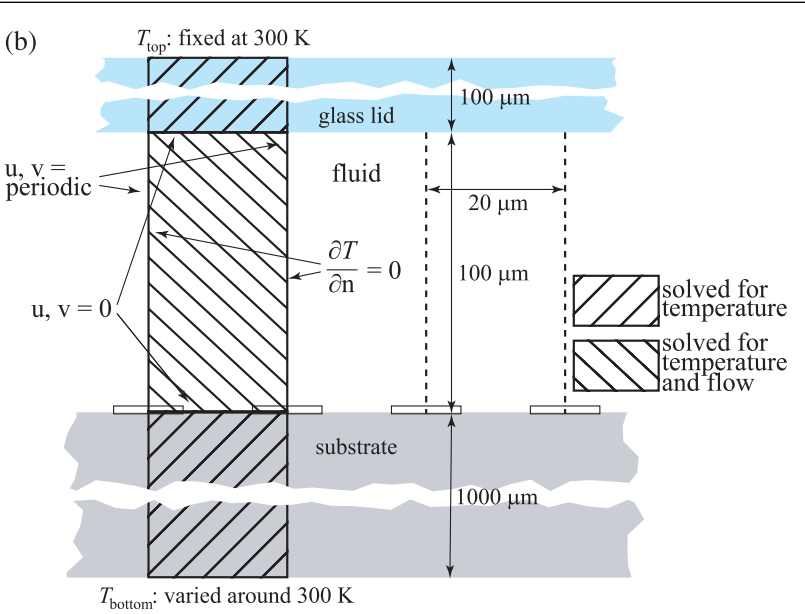

Figure 2. (a) Diagram of the boundary conditions for the electrical problem. (b) Problem space and boundary conditions of the thermal and fluid problems. For the thermal problem, a section of the entire structure is modelled, giving a size of $20 \mu \mathrm{m} \times 1200 \mu \mathrm{m}$, in which the fluid volume is $20 \mu \mathrm{m} \times 100 \mu \mathrm{m}$.

(This figure is in colour only in the electronic version)

them. Heating/cooling through the glass cover and the bottom substrate is included in the model but the electrodes are assumed to be transparent. The top of the glass lid and the base of the substrate are set to be room temperature initially, but can be set to any value to simulate the effects of externally applied temperature gradients.

\subsection{Fluid mechanical model}

The viscosity of the fluid is $8.4 \times 10^{-4} \mathrm{~kg} \mathrm{~m} \mathrm{~s}^{-1}$ at $20^{\circ} \mathrm{C}$. The problem is shown in figure $2(b)$. At the top and bottom the friction gives a no-slip boundary, so the velocity here is set to zero. The left and right borders have periodic boundaries. The force density is found by inserting the obtained values for temperature and field in equation (8), and the Navier-Stokes equation is solved using a penalty method [18].

\section{Results and discussion}

\subsection{Varying the frequency}

For comparison of results the frequency is normalized to $\Omega=\omega \varepsilon / \sigma$, where $\omega=2 \pi f$ is the angular frequency of the applied potential. For frequencies around the charge relaxation time, the induced charge will have a phase lag relative to the electric field vector and the strongest fluid flow occurs when the frequency matches the charge relaxation time of the medium: this happens when $\Omega=1$ [4]. The normalized frequency $\Omega$ is varied logarithmically from $1 \times 10^{-2}$ to $1 \times 10^{2}$. For $\sigma=1 \times 10^{-3} \mathrm{~S} \mathrm{~m}^{-1}$ and $\varepsilon=80 \varepsilon_{0}$, this corresponds to the potential applied to the electrodes being varied in frequency $(f)$ from $2.25 \mathrm{kHz}$ to $22.5 \mathrm{MHz}$; the flow obtains peak velocities for $\Omega=1$, equal to $f=225 \mathrm{kHz}$. To avoid redundancy, the results for a representative selection of frequencies have been chosen to best illustrate the effects.

4.1.1. Glass substrates. Figure 3 shows the flow profile for the glass substrate. In the low-frequency regime $(\Omega \ll 1)$ noticeable vortices are generated in the flow. These vortices are reduced as the frequency approaches $\Omega=1$, where the flow is strongest and unidirectional along the electrode array. At higher frequencies the velocity of the flow reduces but the vortices do not reappear, and the flow pattern remains the same.

4.1.2. Silicon substrates. Figure 4 shows the results for the silicon substrate with the higher thermal conductivity, demonstrating noticeable differences compared to the results for glass. At low frequencies, the vortices are more noticeable than for the glass, with much stronger rotational motion of the fluid over the electrode edges compared to the bulk flow. As for the glass, as the frequency is increased towards $\Omega=1$, the vortices disappear and the flow becomes unidirectional along the electrode array. However, as the frequency is increased further, the velocity decreases as in the case of glass, but smaller vortices reappear over the electrode edges.

In addition, an important point to note is that the direction of the flow is opposite to that of glass in all cases. This result is due to the fact that silicon removes the heat more efficiently than glass, downwards from the system. This has the effect of changing the location of the maximum in the thermal field. In the case of glass, the maximum is elevated above the electrodes in the middle of the fluid, which means that the temperature gradient (and the gradients in conductivity and permittivity) are radial from this position. In the case of silicon, the maximum in the thermal field is pulled down to the surface of the substrate between the electrodes. This subtle difference is thereby pulling the maxima in the temperature down towards the lower surface, moving it to a position with a stronger electric field gradient.

\subsection{Changing the temperature}

The velocity of the fluid can be altered by the application of an external temperature. In this case, the location of the thermal maxima also moves. Therefore, simply reversing the temperature gradient may not lead to a reversal of the fluid flow direction. The effect of the application of an external temperature was examined by changing the temperature difference between top and bottom of the 
(a)

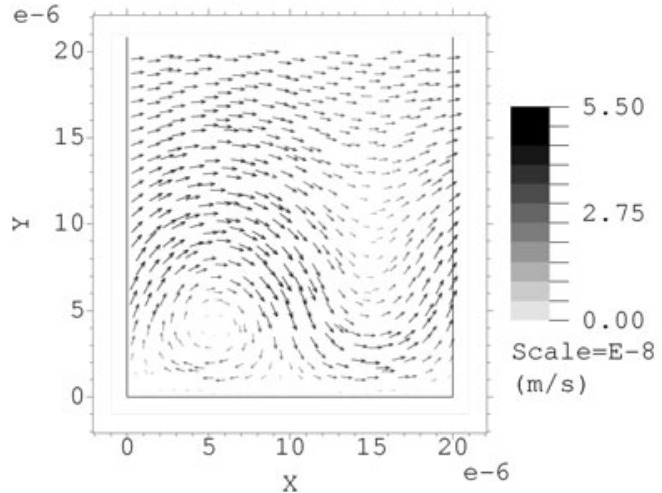

(b)

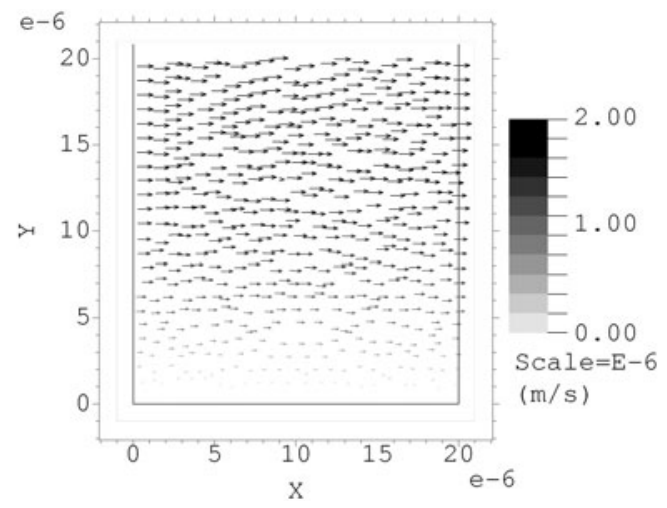

Figure 3. Flow profile on a glass substrate for different frequencies. The volume shown is $20 \mu \mathrm{m} \times 20 \mu \mathrm{m}$ and going from the centre of one electrode to the centre of the next (the lower $20 \mu \mathrm{m}$ of the simulated volume). Conductivity is $\sigma=1 \times 10^{-1} \mathrm{~S} \mathrm{~m}^{-1}$ and $E_{0}=1 \mathrm{~V}$. As expected the flow is strongest in figure $(b)$ where the frequency equals the relaxation frequency $\left(\Omega=1 \times 10^{-2}\right)$. Above the relaxation frequency (not shown) the peak velocity decreases, reaching $3.9 \times 10^{-8} \mathrm{~m} \mathrm{~s}^{-1}$ at $\Omega=1 \times 10^{2}$. However, the vortices do not reappear and the flow pattern continues to look as in figure $(b)(\Omega=1)$.

(a)

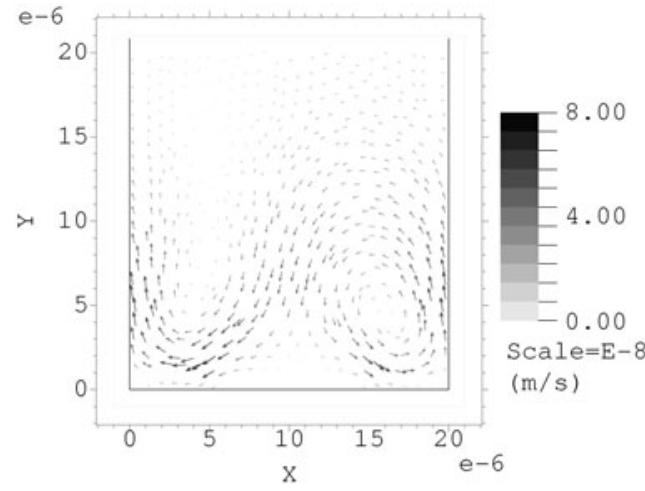

(c)

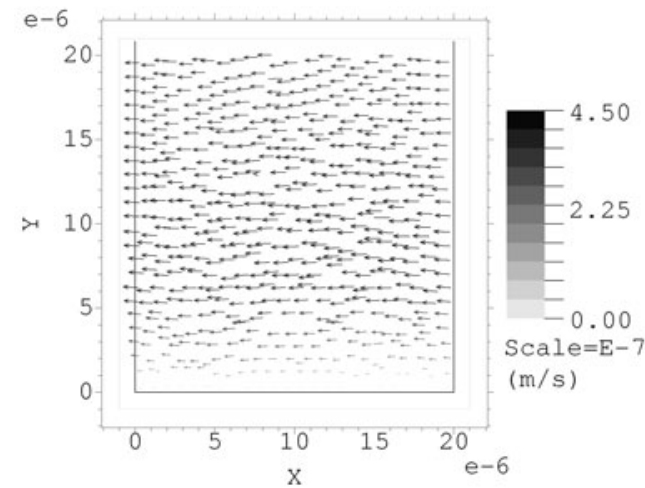

(b)

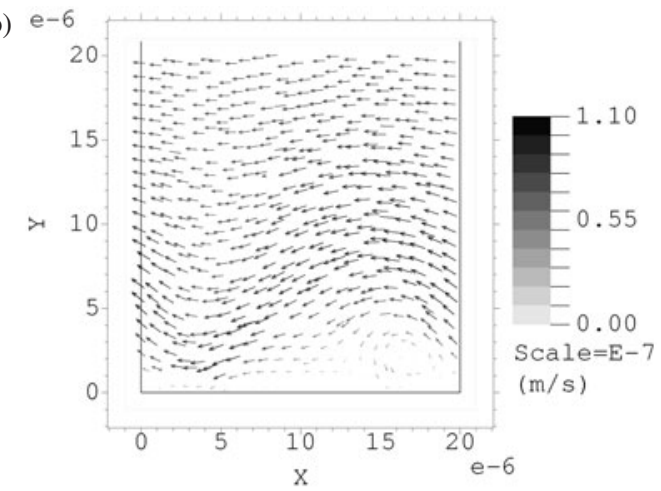

(d)

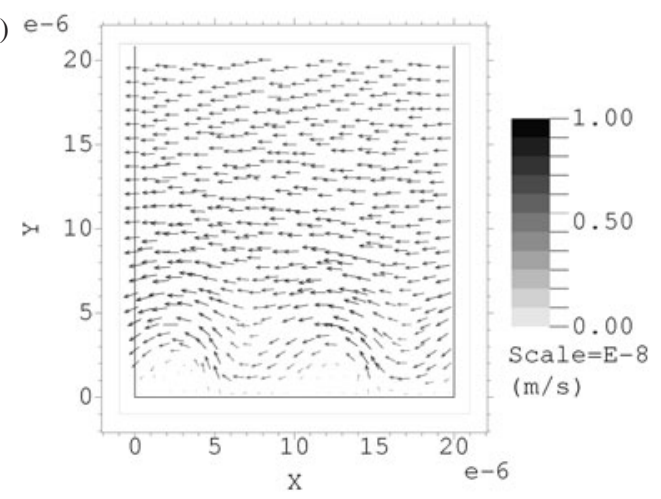

Figure 4. Flow velocity profile on a silicon substrate: $(a) \Omega=1 \times 10^{-2}$, (b) $\Omega=1 \times 10^{-1}$, (c) $\Omega=1$ and $(d) \Omega=1 \times 10^{2}$. Compared to glass the vortices in the flow are visible closed to $\Omega=1$, the maximum velocity is lower, but most noticeable is that the direction of the flow has reversed.

simulated volume: $\Delta T=T_{\text {bottom }}-T_{\text {top }}$. The effect of varying the externally applied temperature on the temperature profile through the fluid volume is shown in figure 5 .

Figures 6 and 7 show how the electrothermal fluid velocity scales with temperature, conductivity and the voltage applied to the electrodes. In each plot, the results from eleven simulations of the fluid velocity are shown. The velocity profiles are obtained by taking the horizontal velocities from the bottom to the top of the fluid volume, along a path on the left border of the boundary in figure $2(b)$. The velocities are different if taken along a different path (e.g. through the centre of the simulation), but the trends between the different cases are the same.

The simulations indicate that the case of no externally applied temperature $(\Delta T=0)$ is a delicate balance, explaining why the difference in thermal conductivity is enough to change the direction of flow for the two different substrates. The results in figures $6(a)$ and $(c)$ show that for low conductivities the velocity responds mainly to the external temperature. Since the electric field is the same regardless of external temperature or substrate, we find from equation (7) that $\nabla T$ controls the overall flow direction. The absolute temperature created 

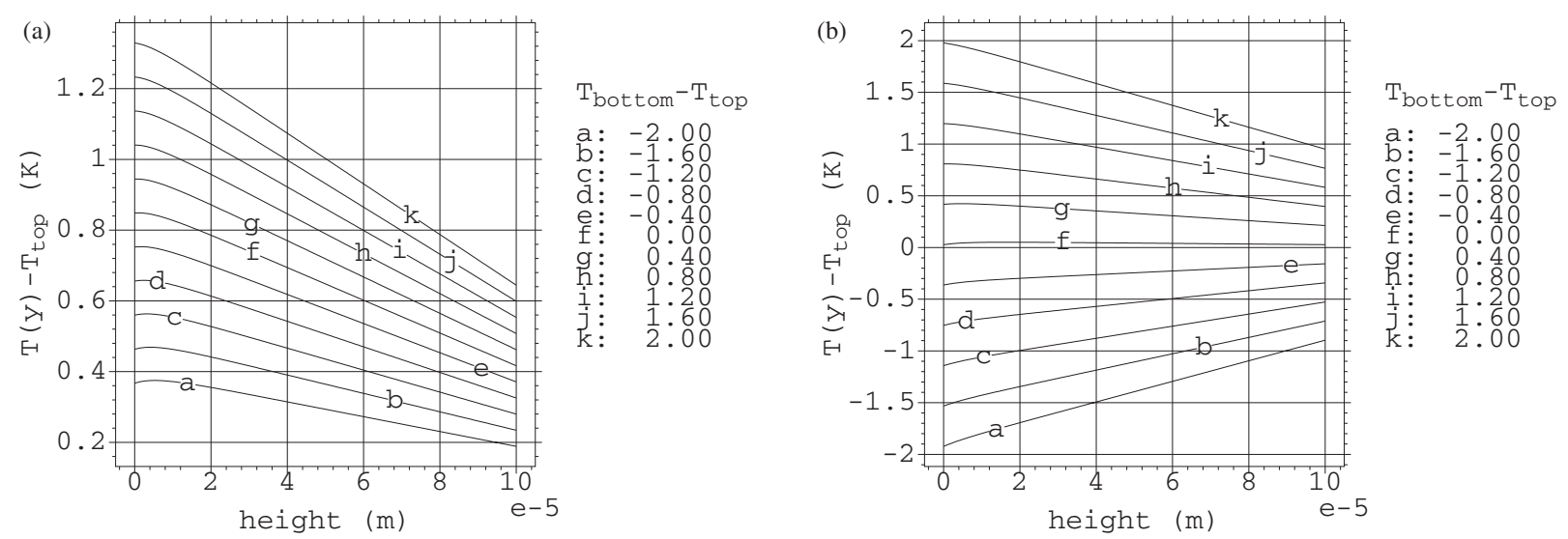

Figure 5. Temperature profile through the fluid as the substrate temperature is varied from $T_{\text {top }}-2 \mathrm{~K}$ to $T_{\text {top }}+2 \mathrm{~K}, \sigma=1 \times 10^{-1} \mathrm{~S} \mathrm{~m}^{-1}$ and $\Omega=1$. The difference between $(a)$ glass and $(b)$ silicon substrate at different temperatures are significant. Since silicon has a high $k$, the temperature at the bottom of the fluid volume is nearly the same as the applied temperature at the base. Glass is insulating and therefore influenced less. There is a maximum in $T$ at different heights, meaning that $\nabla T$ in equation (7) changes sign at different positions in the volume depending on the applied temperature.

(a)

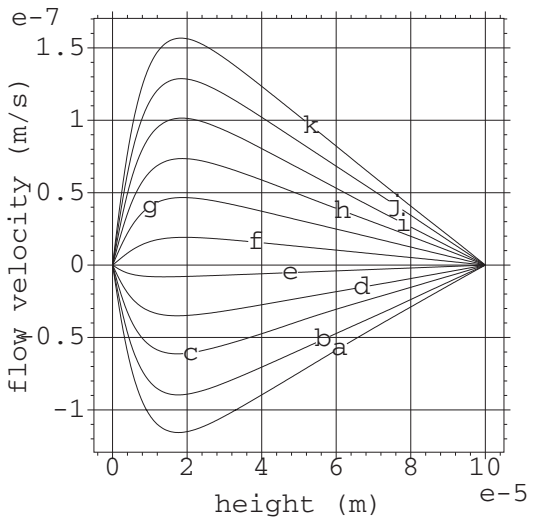

(c)

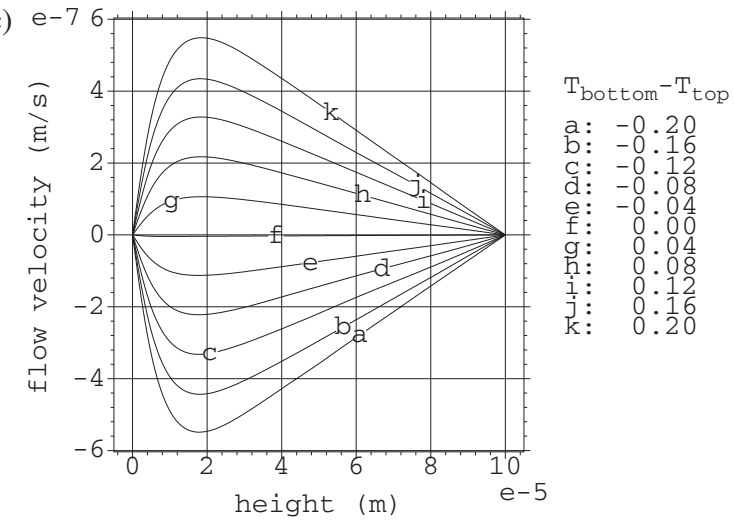

(b)

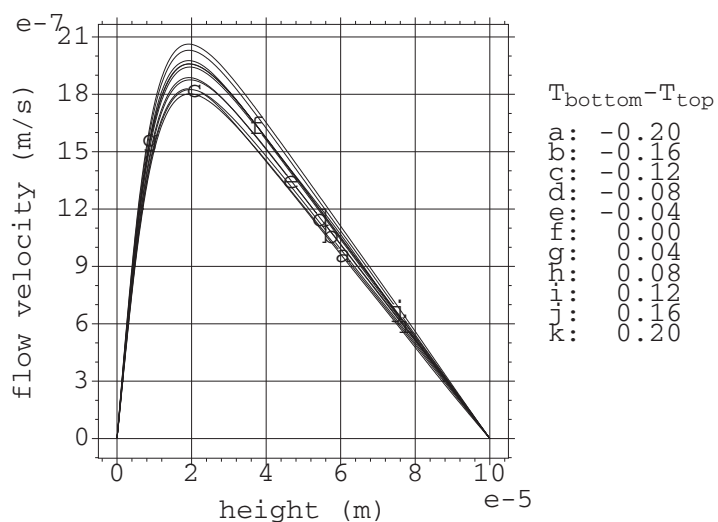

(d)

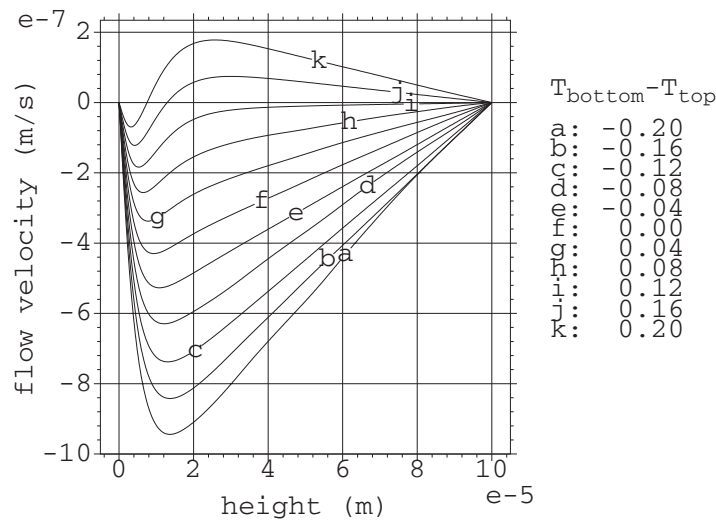

Figure 6. Plots of the velocity along the vertical left edge of the solution space versus height for two different conductivities. Each plot gives the result of 11 simulations, each with a different $\Delta T$. The applied voltage is $E_{0}=1 \mathrm{~V}$ and the frequency is kept at $\Omega=1$. For $\sigma=1 \times 10^{-3} \mathrm{~S} \mathrm{~m}^{-1}$ the flow changes direction as the sign of $\Delta T$ is shifted for both glass $(a)$ and silicon $(c)$. For $\sigma=1 \times 10^{-1} \mathrm{~S} \mathrm{~m}^{-1}$ the overall velocity is increased, but the effect of changing the external temperature is reduced: for glass $(b)$ the velocity is constant and in one direction; for silicon $(d)$, the velocity is in the other direction and forms recirculating patterns as the bottom temperature is increased.

by Joule heating is small (of the order of $0.1 \mathrm{~K}$ ) compared with easily obtainable changes in external temperature, therefore the external temperature is able to impose an overall temperature gradient, thereby moving the maximum in the thermal field.

When verifying simulations experimentally, it is useful to amplify the most striking feature so it can be easily measured, in this case the feature would be that the flow changes direction. This phenomenon is best observed when the fluid velocities are large. Larger fluid velocities can, e.g. be obtained by raising the conductivity of the fluid. However, the ability to control the flow direction by an external temperature is inhibited when the conductivity is increased. This effect can be seen in figures $6(c)$ and $(d)$. Here $\sigma$ is increased two orders 
(a) $e-7$

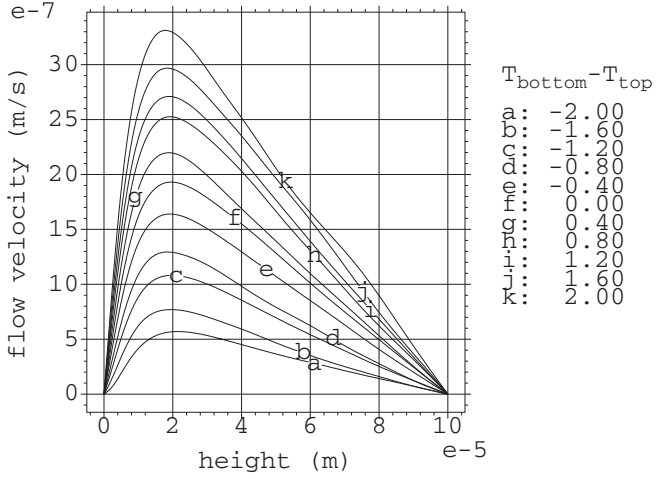

(c)

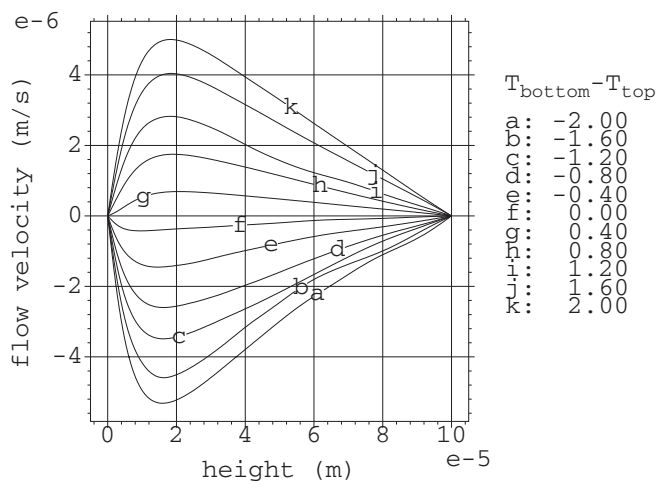

(b)

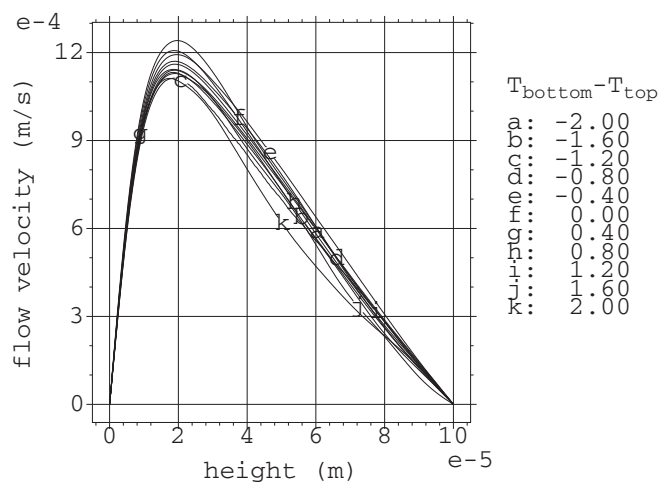

(d)

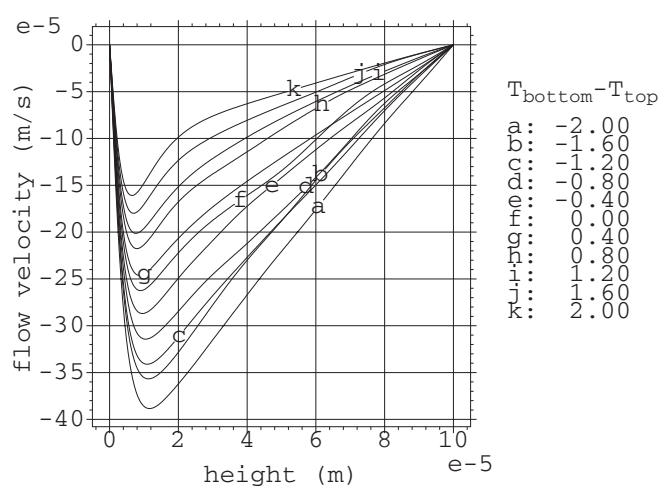

Figure 7. Horizontal flow velocity against height for different $\Delta T$ and applied voltages, with $\sigma=1 \times 10^{-1} \mathrm{~S} \mathrm{~m}{ }^{-1}$ and $\Omega=1$. To examine the flow velocity, the external imposed $\Delta T$ is increased to $\pm 2 \mathrm{~K}$, with the amplitude of the applied voltage $\left(E_{0}\right)$ kept at $1 \mathrm{~V}$ (figures $(a)$ and $(c))$, this results in a large change in the velocities as a function of external temperature. However, when $E_{0}$ is increased as shown in $(b)$ and $(d)$, the internal temperature profile dominates the flow again, and the relative effect of $\Delta T$ decreases.

of magnitude to $1 \times 10^{-1} \mathrm{~S} \mathrm{~m}^{-1}$. Increasing $\sigma$ also fortifies the effect from Joule heating on $\nabla T$ compared to the external imposed temperature. In the case of the glass substrate, this means that the applied temperature of $2 \mathrm{~K}$ is insufficient to alter the direction of the flow, although it is expected that a higher temperature difference will achieve this result.

For the silicon substrate, the higher thermal conductivity means that the external temperature has more influence on $\nabla T$ and, as a result, gives better control of the flow by means of adjusting the external conditions, at a cost of reducing the fluid velocity compared to that on glass substrate. The lower velocity is a result of silicon smoothing the temperature gradient by draining the heat produced by the electric current at a faster rate.

Figure 7 shows how an increased voltage modifies the outcome for the higher conductivity. Increasing the voltage is similar to increasing the conductivity: in both cases the temperature profile in the fluid is affected directly. Again the silicon substrate drains the heat thereby decreasing the flow velocity by an order of magnitude compared to the glass substrate.

Gimsa et al [9] measures peak fluid velocity of approximately $10 \mu \mathrm{m} \mathrm{s}^{-1}$ and $25 \mu \mathrm{m} \mathrm{s}^{-1}$ for a conductivity of $8 \mathrm{mS} \mathrm{m}^{-1}$ and $16 \mathrm{mS} \mathrm{m}^{-1}$, respectively, both with a driving voltage of $25 \mathrm{~V} \mathrm{pk}-\mathrm{pk}$. The gap between successive electrodes was $70 \mu \mathrm{m}$. When simulations is done with the same conditions the average fluid velocity is calculated to be $50 \mu \mathrm{m} \mathrm{s}^{-1}$ and $100 \mu \mathrm{m} \mathrm{s}^{-1}$ for conductivity of $8 \mathrm{mS} \mathrm{m}^{-1}$ and
$16 \mathrm{mS} \mathrm{m}^{-1}$, respectively (both with $\Omega=1$ ). The dependence of the fluid velocity on the driving voltage is a power function [7] and the deviation between measured and simulated velocity corresponds to a factor of two in applied voltage. However, the deviation is probably caused by a combination of factors.

\section{Conclusion}

An electric field on a conducting media will drive a fluid flow through a number of different physical mechanisms. In this paper, the electrothermal driven fluid flow was simulated. The force moving the fluid depends on the temperature gradient, which can change sign a few micrometres above the electrodes, depending on slight external temperatures. The direction of the force depends on the temperature gradient relative to the electric field. In the fluid volume, the temperature profile is governed by fluid conductivity and the applied voltage (to the power 4) from Joule heating. However, if the temperature rise from this is slight compared to obtainable external temperatures, it is possible to move the temperature gradient sufficient to change the flow direction. The effect of imposing an external temperature can be enhanced by selecting a substrate with high thermal conductivity.

Any fluid movement will add to the measured velocity of particles suspended in the fluid, and as such the effect arising from electric fields should be taken into account both when measuring particle velocities. In the case of a travelling 
wave, the direction of the fluid relative to the field can change depending on the external temperature profile, either deliberately or accidentally applied.

\section{References}

[1] Shapiro H M 1995 Practical Flow Cytometry 3rd edn (New York: Wiley)

[2] Pohl H A 1978 Dielectrophoresis (Cambridge: Cambridge University Press)

[3] Jones T B 1995 Electromechanics of Particles (Cambridge: Cambridge University Press)

[4] Morgan H and Green N G 2003 AC Electrokinetics: Colloids and Nanoparticles (Herts: Research Studies Press)

[5] Burt J P H, Pethig R and Talary M S 1998 Microelectrode devices for manipulating and analysing bioparticles Trans. Inst. Meas. Control 20 82-90

[6] Morgan H, Izquierdo A G, Bakerwell D, Green N G and Ramos A 2001 The dielectrophoretic and travelling wave forces generated by interdigitated electrode arrays: analytical solution using fourier series J. Phys. D: Appl. Phys. 34 1553-61

[7] Müller T, Arnold W M, Schnelle T, Hagedorn R, Fuhr G and Zimmermann U 1993 A traveling wave micropump for aqueous solutions: comparison of $1 \mathrm{~g}$ and $\mu \mathrm{g}$ results Electrophoresis 14 764-72

[8] Fuhr G, Schnelle T and Wagner B 1994 Travelling wave driven microfabricated electrohydrodynamic pumps for liquids J. Micromech. Microeng. 4 217-26
[9] Gimsa J, Eppmann P and Prüger B 1997 Introducing phase analysis light scattering for dielectric characterization: measurement of travelling wave pumping Biophys. $J$. 73 3309-16

[10] Ramos A, Morgan H, Green N G and Castellanos A 1998 AC electrokinetics: a review of forces in microelectrode structures J. Phys. D: Appl. Phys. 31 2338-53

[11] Castellanos A, Ramos A, González A, Green N G and Morgan H 2003 Electrohydrodynamics and dielectrophoresis in microsystems: scaling laws $J$. Phys. $D$ : Appl. Phys. 36 2584-97

[12] Stratton J A 1941 Electromagnetic Theory (New York: McGraw-Hill)

[13] Melcher J R 1981 Continuum Electromechanics (Cambridge: MIT Press)

[14] Landau L D and Colburn J C 1959 Capillary Electrophoresis (New York: Academic)

[15] Castellanos A 1998 Electrohydrodynamics (Wien, New York: Springer)

[16] Green N G, Ramos A, González A, Castellanos A and Morgan H 2001 Electrothermally induced fluid flow on microelectrodes J. Electrostat. 53 71-87

[17] Lide D R 1994 CRC Handbook of Chemistry and Physics 74th edn (London: CRC Press)

[18] Backstrom G 1999 Fluid Dynamics by Finite Element Analysis (Lund: Studentlitteratur)

[19] FlexPDE v3.03 PDE solutions, http://www.pdesolutions.com/

[20] Green N G, Ramos A and Morgan H 2002 Numerical solution of the dielectrophoretic and travelling wave forces for interdigitated electrode arrays using the finite element method J. Electrostat. 56 235-54 